\title{
Solid lipid nanoparticles loaded with iron to overcome barriers for treatment of iron deficiency anemia
}

This article was published in the following Dove Press journal:

Drug Design, Development and Therapy

8 January 2015

Number of times this article has been viewed

\author{
Khaled Mohamed Hosny,2 \\ Zainy Mohammed Banjar ${ }^{3}$ \\ Amani $\mathrm{H} \mathrm{Hariri}{ }^{4}$ \\ Ali Habiballah Hassan ${ }^{5}$ \\ 'Department of Pharmaceutics \\ and Industrial Pharmacy, Faculty \\ of Pharmacy, King Abdulaziz \\ University, Jeddah, Saudi Arabia; \\ ${ }^{2}$ Department of Pharmaceutics \\ and Industrial Pharmacy, Faculty \\ of Pharmacy, Beni Suef University, \\ Beni Suef, Egypt; ${ }^{3}$ Department \\ of Clinical Biochemistry, Faculty \\ of Medicine, King Abdulaziz University, \\ Jeddah, Saudi Arabia; ${ }^{4}$ Consultant \\ Obstetrics and Gynecology, Hera \\ General Hospital, Makkah, Saudi \\ Arabia; ${ }^{5}$ Department of Orthodontics, \\ Faculty of Dentistry, King Abdulaziz \\ University, Jeddah, Saudi Arabia
}

Correspondence: Khaled Mohamed Hosny PO Box 80260, Department of Pharmaceutics and industrial pharmacy, Faculty of Pharmacy, King Abdulaziz University, Jeddah 21589 , Saudi Arabia Tel +966592722634

Email kmhomar@kau.edu.sa

\begin{abstract}
According to the World Health Organization, 46\% of the world's children suffer from anemia, which is usually treated with iron supplements such as ferrous sulfate. The aim of this study was to prepare iron as solid lipid nanoparticles, in order to find an innovative way for alleviating the disadvantages associated with commercially available tablets. These limitations include adverse effects on the digestive system resulting in constipation and blood in the stool. The second drawback is the high variability in the absorption of iron and thus in its bioavailability. Iron solid lipid nanoparticles (Fe-SLNs) were prepared by hot homogenization/ultrasonication. Solubility of ferrous sulfate in different solid lipids was measured, and effects of process variables such as the surfactant type and concentration, homogenization and ultrasonication times, and charge-inducing agent on the particle size, zeta potential, and encapsulation efficiency were determined. Furthermore, in vitro drug release and in vivo pharmacokinetics were studied in rabbits. Results indicated that Fe-SLNs consisted of 3\% Compritol 888 ATO, 1\% Lecithin, 3\% Poloxamer 188, and $0.2 \%$ dicetylphosphate, with an average particle size of $25 \mathrm{~nm}$ with $92.3 \%$ entrapment efficiency. In vivo pharmacokinetic study revealed more than fourfold enhanced bioavailability. In conclusion, Fe-SLNs could be a promising carrier for iron with enhanced oral bioavailability.
\end{abstract}

Keywords: iron, anemia, solid lipid nanoparticles, bioavailability, Compritol 888 ATO

\section{Introduction}

Iron deficiency anemia is a common form of anemia that occurs in conditions of iron loss and/or in case of problems in iron absorption from the dietary intake. ${ }^{1}$ Iron deficiency is the most common cause of anemia worldwide, accounting for about half of all anemia cases. ${ }^{2}$ Estimates of iron deficiency worldwide range vary widely, but the number almost certainly exceeds one billion persons globally. There are many diseases that might lead to iron deficiency anemia, including intestinal parasitic infection, malarial infections, gastric ulcer, duodenal ulcer, and gastrointestinal cancer. ${ }^{3}$

According to the World Health Organization, 46\% of the world's children are anemic, the greatest percentage being in developing countries. About $56 \%$ of pregnant women in these countries are anemic. ${ }^{3}$ In the developed world, about $20 \%$ of all women of childbearing age have iron deficiency anemia compared with only $3 \%$ of adult men. ${ }^{4-7}$

Iron deficiency anemia is usually treated by the use of iron supplements. The most famous and commercially available supplements in the market are ferrous sulfate, ferrous fumarate, and ferrous gluconate. However, all these iron salts have two important disadvantages. The first one is that all are known to affect the gastrointestinal tract 
(GIT) and to result in many side effects. The most common side effects include constipation, darkened or green stools, diarrhea, nausea, stomach upset, and temporary staining of the teeth. In addition, sometimes severe reactions may occur, including allergic reactions, black and tarry stools, blood or streaks of blood in the stool, fever, and vomiting with continuing sharp stomach pain. The second disadvantage is the high variability in iron absorption from the GIT and hence variability in the bioavailability. Several researches have been carried out using iron in different dosage forms as intravenous injection for the treatment of anemia. ${ }^{8,9}$

It is reported that even the same iron salt developed by different pharmaceutical companies show different bioavailabilties. ${ }^{10}$ For example, the bioavailability of iron from ferrous sulfate varies considerably among studies. The efficiency of converting iron in ferrous sulfate into hemoglobin ranged from $33 \%$ to $80 \% .^{11}$

Consequently, there is a need for developing a new, stable, highly soluble system in which iron is soluble in the different $\mathrm{pH}$ conditions inside the GIT and which enhances the permeability of iron through the GIT. In addition, the system should result in a more reproducible bioavailability.

Nanotechnology deals with the utilization of materials at the nanoscale, and research in the field of nanotechnology is attracting countries and large companies to invest massively in this fast growing area. Materials at the nanometer scale exploit their novel physical, chemical, and biological properties that can change or enhance drug properties.

Treatment of anemia with novel drug delivery systems has been previously mentioned in several publications; one of them used magnetite and folate nanoparticles (NPs) ${ }^{12}$ and another prepared iron as polymeric NPs. ${ }^{13,14}$

Studies on the GIT mucosal absorption of particulate matter delivered through the oral route showed that particle size is a critical factor for absorption. ${ }^{15}$ There are three possible routes of NP absorption: transcellular transport, paracellular transport (particles less than $500 \mathrm{~nm}$ ), and uptake of NPs by the gut $\mathrm{M}$ cells. ${ }^{16}$ Various factors control drug release from NPs, including the type of polymer, its physical and chemical properties, the presence of stabilizers, and type of formulation. ${ }^{17}$

Solid lipid nanoparticles (SLNs) have been reported as an alternative drug delivery system to liposomes, emulsions, microparticles, and polymeric nanoparticulate systems. ${ }^{18}$ This novel colloidal drug carrier system enables various application routes such as oral, topical, parenteral, ophthalmic, and rectal. ${ }^{19}$ SLNs offer numerous advantages over other colloidal systems, such as the possibility of large-scale manufacture and enhancement of absorption of various drugs (hydophilic or lipophilic) where the absorption of NPs takes place by several mechanisms, namely via the Peyer's patches either by the intracellular pathway or by paracellular uptake. ${ }^{20}$ Furthermore, delivery via SLNs avoids to a large extent the hepatic first-pass effect due to absorption into lymphatic circulation, which bypasses the liver. ${ }^{21}$ Controlled and sustained drug delivery and drug targeting are also achieved. ${ }^{22}$ SLNs can be prepared by using several methods: high-pressure homogenization (hot and cold homogenization), solvent evaporation, ${ }^{23}$ solvent emulsification-diffusion, ${ }^{24}$ microemulsion, double emulsion, ${ }^{25}$ and high-shear homogenization followed by ultrasonication. ${ }^{26}$ In the homogenization/ultrasonication method, the main parameters that affect the particle size of the SLN formulation are the type of the lipid, type of the emulsifier, their concentrations, and the homogenization and sonication times. ${ }^{27}$

The objective of this work was to utilize nanotechnology to formulate ferrous sulfate into SLNs and study the effect of different process variables on the particle size, encapsulation efficiency (EE), and stability of theses NPs, in order to provide a solution to the major problems associated with the marketed iron supplement tablets.

\section{Experimental Materials}

Ferrous sulfate was received as a kind gift from Sandoz Ltd. (Surrey, UK). Compritol 888 ATO, Precirol ATO 5, Labrasol, Gelucire 44/14, and Sedefos were obtained from Gattefosse (Nanterre Sedex, France). Plurol stearique, cholesteryl stearate, and phosphatidylcholine were a kind gift from Nikko chemicals Co., Ltd (Tokyo, Japan). Stearylamine (SA) and dihexadecyl hydrogen phosphate (DHP) were purchased from Fluka Chemical Company (Buchs, Switzerland). Poloxamer 188 was purchased from Sigma-Aldrich (St Louis, MO, USA). Ferrous sulfate marketed tablet (Ferrous sulfate tablet $^{\circledR}$ ) was purchased from Sandoz Ltd. All other reagents and chemicals were of analytical grade.

\section{Methods}

\section{Measurement of ferrous sulfate solubility in lipids}

The method depends on measurement of the partitioning behavior of iron in various lipids (Compritol 888 ATO, Precirol ATO 5, Plurol stearique, and cholesteryl stearate). One-hundred milligrams of ferrous sulfate was dispersed in a mixture of melted lipid $(1 \mathrm{~g})$ at $80^{\circ} \mathrm{C}$ and $1 \mathrm{~mL}$ of hot distilled water and shaken for 30 minutes in a hot water bath. The aqueous phase was separated after cooling and 
centrifugation at 15,000 rpm for 30 minutes with the help of a syringe and analyzed for iron content by atomic absorption spectrometry at $248.3 \mathrm{~nm}$.

\section{Preparation of ferrous sulfate solid lipid nanoparticles}

Iron solid lipid nanoparticles (Fe-SLNs) were prepared by the hot emulsification/ultrasonication method. ${ }^{28}$ According to the result of the partitioning test, Compritol 888 ATO was chosen as a solid lipid for the formulation of Fe-SLNs. Ferrous sulfate (2\%), Compritol 888 ATO (3\%), and lecithin $(1 \%)$ were dissolved in a $25-\mathrm{mL}$ mixture of chloroform and methanol (1:1). The organic solvents were then completely removed using a rotary evaporator. The drug-embedded lipid layer was melted by heating at $80^{\circ} \mathrm{C}$, and the aqueous phase was prepared by dissolving one of the following surfactants, namely Cremophor, Gelucire, Poloxamer 188, Labrasol, or Sedefos, in double-distilled water (sufficient to produce $25 \mathrm{~mL}$ of the preparation) and heated to the same temperature of the molten lipid phase. The hot aqueous phase was added to the molten lipid phase, and homogenization was carried out at $10,000 \mathrm{rpm}$ for 3 minutes, maintaining the temperature at $80^{\circ} \mathrm{C}$. Coarse, hot oil-in-water emulsion was obtained, which was then ultrasonicated using a probe sonicator. Fe-SLNs were obtained by allowing the hot nanoemulsion to cool to room temperature. SA or DHP was used as the surface charge modifier of the SLNs, which was incorporated with the lipids in some formulations. Different formulations were prepared by varying the critical process variables, as described in the following section.

\section{Formulation design}

SLNs were prepared by varying the surfactant type (ST), surfactant concentration (SC), lecithin concentration (LC), homogenization time (HT), and ultrasonication time (UT) as follows:

a) $\mathrm{STs}=($ Cremophor, Gelucire 44/14, Poloxamer 188, Labrasol, and Sedefos); while HT $=3$ minutes, $\mathrm{UT}=3$ minutes, and $\mathrm{SC}=3 \%(\mathrm{w} / \mathrm{v})$;

b) $\mathrm{SC}(0.5,1,2,3$, and $4 \%, \mathrm{w} / \mathrm{v})$; while $\mathrm{HT}=3$ minutes, $\mathrm{UT}=3$ minutes, and $\mathrm{ST}=$ Poloxamer 188 ;

c) $\mathrm{HT}(1,3,5$, and 10 minutes); while UT $=1$ minute, $\mathrm{SC}=3 \%$ $(\mathrm{w} / \mathrm{v})$, and $\mathrm{ST}=$ Poloxamer 188 ;

d) $\operatorname{UT}(1,3,5$, and 7); while $\mathrm{HT}=3$ minutes, $\mathrm{SC}=3 \%(\mathrm{w} / \mathrm{v})$, and $\mathrm{ST}=$ Poloxamer 188;

e) $\mathrm{LC}(0 \%$ or $1 \%)$; while $\mathrm{HT}=3$ minutes, $\mathrm{UT}=3$ minutes, $\mathrm{SC}=3 \%(\mathrm{w} / \mathrm{v})$, and $\mathrm{ST}=$ Poloxamer 188 ; f) Charge inducing agent (SA as a positive charge inducing agent, DHP as a negative charge inducing agent, or neutral without any charge inducing agent); while HT $=3$ minutes, UT $=3$ minutes, $\mathrm{SC}=3 \%(\mathrm{w} / \mathrm{v})$, and $\mathrm{ST}=$ Poloxamer 188.

\section{Measurement of particle size and zeta potential}

Fe-SLNs dispersions were appropriately diluted with ultrapurified water before measurement. The hydrodynamic diameter and zeta potential of the resulting dispersion were determined by dynamic light scattering using a Zetatrac machine (Microtrac Inc, PA, USA).

\section{Measurement of entrapment efficiency}

The entrapment efficiency of ferrous sulfate within Fe-SLNs was determined by measuring the concentration of free drug in the dispersion medium. Ultracentrifugation was carried out using Centrisart, which consists of a filter membrane (molecular weight cut-off 20,000 Da) at the base of the sample recovery chamber. Approximately $1 \mathrm{~mL}$ of undiluted sample was placed in the outer chamber, and the sample recovery chamber was placed on the top of the sample. The unit was centrifuged at 5,000 rpm for 30 minutes. The Fe-SLNs along with encapsulated drug remained in the outer chamber and the aqueous phase moved into the sample recovery chamber through the filter membrane. The amount of iron in the aqueous phase was calculated by atomic absorption spectrometry at $248.3 \mathrm{~nm}$.

The EE was calculated as

$$
\mathrm{EE}(\%)=100-(\mathrm{Fs} / \mathrm{Ts} \times 100)
$$

where Fs = soluble free drug, and Ts = initial amount of drug added during preparation of SLNs.

\section{Drug release study}

Drug release study was performed following the dialysis bag method. ${ }^{29}$ The Fe-SLN suspension was placed in dialysis bags with 12-kDa molecular weight cut-off (Sigma) and immersed in $500 \mathrm{~mL} 0.1 \mathrm{~N} \mathrm{HCl}$ at $37^{\circ} \mathrm{C}$ in a dissolution apparatus with a paddle rotating at $50 \mathrm{rpm}$. An aliquot of sample $(5 \mathrm{~mL})$ was taken from the dissolution medium after 0.25 hours, 0.5 hours, 1 hours, 1.5 hours, 2.0 hours, and 2.5 hours. The $\mathrm{pH}$ of the dissolution medium was raised to 7.4, and samples were then taken after 3 hours, 4 hours, 6 hours, 8 hours, 12 hours, and 24 hours and analyzed for iron content by atomic absorption spectrometry at $248.3 \mathrm{~nm}$. 


\section{Stability study}

Stability of the prepared Fe-SLN formulations was tested with heating and cooling cycles. Three freeze-thaw cycles $\left(-20^{\circ} \mathrm{C}\right.$ and $\left.+25^{\circ} \mathrm{C}\right)$ were performed on these formulations. Then the particle size and zeta potential were measured. SLNs that showed good dispersion stability test were selected for in vivo pharmacokinetic study.

\section{In vivo pharmacokinetic study}

In vivo drug absorption study was conducted according to the institutional guidelines of the Animal Ethics Committee, Faculty of Pharmacy, King Abdulaziz University. Twelve albino male rabbits, weighing between $2 \mathrm{~kg}$ and $2.5 \mathrm{~kg}$, were used in this study. Based on the results of in vitro and stability studies, negatively charged Fe-SLN formulation, composed of $200 \mathrm{mg}$ ferrous sulfate, $300 \mathrm{mg}$ Compritol 888 ATO as a solid lipid, $300 \mathrm{mg}$ Poloxamer 188 as surfactant, $100 \mathrm{mg}$ lecithin, and $20 \mathrm{mg}$ DHP, prepared by 3-minute homogenization followed by 3-minute ultrasonication method, was chosen for the animal studies.

The rabbits were kept in a fasting condition for 24 hours before the experiment commenced, and they were divided into two groups, each containing six animals. Group I was treated with ferrous sulfate tablet (as a control), which was crushed and suspended in $5 \mathrm{~mL}$ of saline. The equivalent volume containing $10 \mathrm{mg} / \mathrm{kg}$ was administered orally via gastric intubation for each fasted rabbit. Group II was treated orally with Fe-SLN formulation, which was suspended in $5 \mathrm{~mL}$ of saline, and an equivalent volume containing $10 \mathrm{mg} / \mathrm{kg}$ was administered orally via gastric intubation for each rabbit in the group.

Blood samples $(2 \mathrm{~mL})$ were collected before the administration of the drug and again at different time intervals ( 0.5 hours, 0.75 hours, 1 hours, 1.5 hours, 2 hours, 3 hours, 4 hours, 6 hours, 9 hours, 12 hours, and 24 hours) after the administration of the drug. The obtained serum samples were deep-frozen at $-20^{\circ} \mathrm{C}$, pending high-performance liquid chromatography (HPLC) analysis. The plasma analysis of ferrous sulfate was performed using a well-validated HPLC method described by Dias et al after liquid-liquid extraction. ${ }^{30}$

\section{Pharmacokinetic analysis}

The maximum plasma concentration $\left(C_{\max }\right)$ and time required to reach maximum plasma level $\left(T_{\max }\right)$ were calculated using the plasma concentration versus time curve in the WinNonlin ${ }^{\mathrm{TM}}$ Nonlinear Estimation Program. One-way analysis of variance (ANOVA) was employed to assess the significance of the difference between the $T_{\max }, C_{\max }$, and $\mathrm{AUC}_{0-\infty}$ data of ferrous sulfate obtained from the control tablet and Fe-SLN formulations $(P \leq 0.05)$ using the SPSS program. ${ }^{31}$ The $\mathrm{AUC}_{0-24}$ was calculated using the linear trapezoidal rule. The $\mathrm{AUC}_{0-\infty}$ and the relative bioavailability $\left(\mathrm{BA}_{\mathrm{R}}\right)$ of the SLNs were also calculated by using the following equation ${ }^{32}$ :

Relative bioavailability $\left(\mathrm{BA}_{\mathrm{R}}\right)=\left[\mathrm{AUC}_{\mathrm{SLNs}} \times\right.$ Dose $\left._{\text {Tablet }}\right] /$ $\left[\mathrm{AUC}_{\text {Tablet }} \times\right.$ Dose $\left._{\mathrm{SLNs}}\right]$

\section{Results}

\section{Screening of lipid phase}

For the selection of the lipid core, solubility of ferrous sulfate in lipids was evaluated by measuring the ratio of the amount of iron in lipid to the amount of iron in aqueous phase. The results obtained were $21.23 \pm 1.14,15.53 \pm 1.33,62.11 \pm 3.44$, and $46.61 \pm 3.03$ for Plurol stearique, cholesteryl stearate, Compritol 888 ATO, and Precirol ATO 5, respectively. The highest partition coefficient was observed with Compritol 888 ATO, which was selected as a core lipid for the preparation of Fe-SLNs.

\section{Preparation and characterization of Fe-SLNs}

Hot homogenization followed by ultrasonication was found to be an efficient, simple, and quick method to produce Fe-SLNs. In order to optimize the preparation of the SLN, several formulation variables were studied. The effect of the different process variables on particle size, zeta potential, entrapment efficiency is presented in Table 1.

\section{Drug release study}

According to the results of particle size and entrapment efficiency, the release of the best Fe-SLN formula prepared from $200 \mathrm{mg}$ ferrous sulfate, $300 \mathrm{mg}$ Compritol 888 ATO as a solid lipid, $300 \mathrm{mg}$ Poloxamer 188 as surfactant, $100 \mathrm{mg}$ lecithin, and $20 \mathrm{mg}$ DHP, prepared by 3-minute homogenization followed by 3-minute ultrasonication was compared with the release of pure drug, and Fe-SLNs displayed a biphasic drug release pattern with a burst release within 30 minutes, followed by sustained release afterward, as shown in Figure 1.

\section{Thermodynamic stability of the Fe-SLNs}

Stability test showed that there was a statistically nonsignificant difference in the $\mathrm{EE} \%$ or in particle size with charged Fe-SLNs after being subjected to three freeze-thaw cycles. However, the changes were significant in the case of neutral SLNs in which the particle size increased from $26 \mathrm{~nm}$ to $113 \mathrm{~nm}$. These results indicated a good stability for charged SLNs in comparison with neutral ones. 
Table I Particle size (diameter), zeta potential, and entrapment efficiency measurements of the Fe-SLNs prepared by varying ST, SC, HT, UT, LC, and charge inducing agent (data represented as mean \pm SD)

\begin{tabular}{|c|c|c|c|c|}
\hline Variable & & Particle size (nm) & Zeta potential $(\mathrm{mV})$ & Entrapment efficiency (\%) \\
\hline \multirow[t]{5}{*}{ ST } & Cremophor & $40.6 \pm 3.22$ & $8.22 \pm 1.11$ & $75.43 \pm 3.42$ \\
\hline & Labrasol & $34.76 \pm 2.67$ & $7.13 \pm 1.32$ & $82.14 \pm 4.14$ \\
\hline & Poloxamer I88 & $26.11 \pm 1.35$ & $8.13 \pm 1.02$ & $92.30 \pm 3.11$ \\
\hline & Gelucire 44/I 4 & $45.12 \pm 3.11$ & $7.31 \pm 0.82$ & $71.26 \pm 2.82$ \\
\hline & Sedefos & $60.11 \pm 4.02$ & $8.66 \pm 0.97$ & $65.11 \pm 3.66$ \\
\hline \multirow[t]{5}{*}{ SC } & $0.5 \%$ & $1,015 \pm 108$ & $8.62 \pm 1.25$ & $81.06 \pm 5.01$ \\
\hline & $1 \%$ & $705 \pm 90$ & $8.18 \pm 1.67$ & $83.72 \pm 3.26$ \\
\hline & $2 \%$ & $232 \pm 54$ & $8.25 \pm 1.04$ & $88.33 \pm 4.12$ \\
\hline & $3 \%$ & $26.11 \pm 1.35$ & $8.13 \pm 1.02$ & $92.30 \pm 3.11$ \\
\hline & $4 \%$ & $24.22 \pm 1.22$ & $8.23 \pm 1.41$ & $85.22 \pm 3.14$ \\
\hline \multirow[t]{4}{*}{ HT (minutes) } & 1 & $|43.32 \pm 4.2|$ & $8.25 \pm 1.72$ & $88.72 \pm 3.42$ \\
\hline & 3 & $95.01 \pm 4.92$ & $8.07 \pm 1.22$ & $90.30 \pm 3.45$ \\
\hline & 5 & $93.11 \pm 5.29$ & $7.99 \pm 1.14$ & $91.58 \pm 3.22$ \\
\hline & 10 & $90.06 \pm 3.98$ & $8.52 \pm 1.09$ & $92.17 \pm 2.89$ \\
\hline \multirow[t]{4}{*}{ UT (minutes) } & 1 & $95.01 \pm 4.92$ & $8.07 \pm 1.22$ & $90.30 \pm 3.45$ \\
\hline & 3 & $26.11 \pm 1.35$ & $8.13 \pm 1.02$ & $92.30 \pm 3.11$ \\
\hline & 5 & $24.62 \pm 2.45$ & $8.43 \pm 1.19$ & $92.57 \pm 3.18$ \\
\hline & 7 & $24.2 I \pm 2.24$ & $8.34 \pm 0.98$ & $90.13 \pm 2.82$ \\
\hline \multirow[t]{2}{*}{ LC } & $0 \%$ & $45.24 \pm 2.91$ & $8.11 \pm 0.94$ & $76.11 \pm 3.10$ \\
\hline & $1 \%$ & $26.11 \pm 1.35$ & $8.13 \pm 1.02$ & $92.30 \pm 3.11$ \\
\hline \multirow[t]{2}{*}{ Charge inducing agent } & Stearylamine & $27.20 \pm 3.43$ & $16.04 \pm 2.11$ & $83.01 \pm 2.16$ \\
\hline & Dicetyl phosphate & $25.01 \pm 1.21$ & $-10.13 \pm 0.62$ & $92.30 \pm 3.11$ \\
\hline
\end{tabular}

Abbreviations: Fe-SLNs, iron solid lipid nanoparticles; ST, surfactant type; SC, surfactant concentration; HT, homogenization time; UT, ultrasonication time; LC, Lecithin concentration; SD, standard deviation.

\section{In vivo pharmacokinetic study}

There was a statistically significant difference in the $T_{\max }, C_{\max }$, and $\mathrm{AUC}_{0-\infty}$ data between the Fe-SLNs and the marketed tablet $(P \leq 0.05)$ (Table 2 and Figure 2). The mean $\mathrm{AUC}_{0-\infty}$ for the marketed tablet was significantly different from that for the $\mathrm{Fe}$ SLNs. These results confirmed that the formulation of iron into SLNs increased its bioavailability by more than fourfold.

\section{Discussion}

Solubility of drugs in solid lipid is known to be an important precondition to obtain sufficient entrapment. ${ }^{33}$ In order

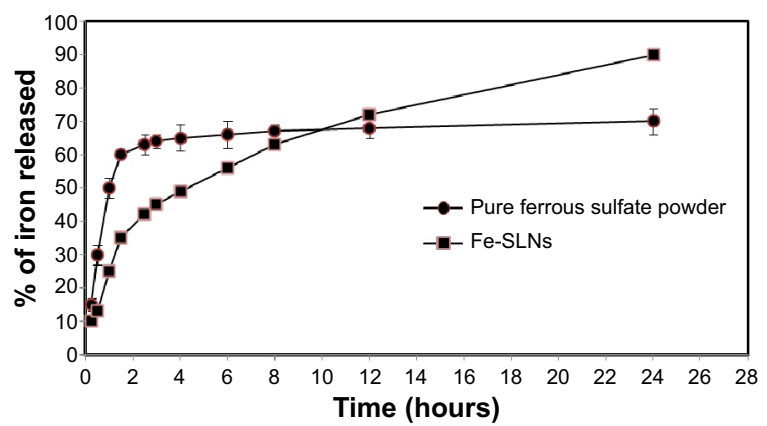

Figure I In vitro release profile of iron from Fe-SLNs and pure ferrous sulfate powder.

Abbreviation: Fe-SLNs, iron solid lipid nanoparticles. to optimize the preparation of Fe-SLNs, solubility studies were carried out by measuring the partitioning between different solid lipids and water phases. It was found that iron had better solubility in Compritol 888 ATO, which contains in its structure a longer chain fatty alcohol than the other lipids used, and led to the creation of a less ordered solid lipid matrix leaving enough space to accommodate the drug molecule. ${ }^{34}$

The presence of lecithin in SLNs is essential in the lipid matrix, as it contains spaces where the drug can be localized and reduces the escape of the drug into the external phase due to the hydrophilic group contained in the lecithin structure. This accounts for the increase in entrapment efficiency seen when compared with SLNs formulated without lecithin. ${ }^{35}$

The reduction in particle size during the production of SLNs leads to an increase in the attraction between the particles, which increases the surface tension at the interface, leading to physical instability. ${ }^{36}$ The incorporation of surfactant in the formulation imparts a repulsive force between the NPs and reduces the surface energy and the interfacial tension of the system. Hence, the selection of the type and concentration of surfactant is important for the physical characteristics and stability of SLNs. ${ }^{37}$ 
Table 2 Pharmacokinetic parameters of iron after administration of $10 \mathrm{mg} / \mathrm{kg}$ oral Fe-SLNs and $10 \mathrm{mg} / \mathrm{kg}$ of marketed tablet $(\mathrm{n}=6$ )

\begin{tabular}{llllll}
\hline Formulation & $T_{\max }(\mathbf{h})$ & $\boldsymbol{C}_{\max }(\mathrm{ng} / \mathrm{mL})$ & $\mathbf{A U C}_{0-\mathrm{t}}(\mathbf{n g ~ h} / \mathrm{mL})$ & $\mathbf{K}_{\left(\mathbf{h}^{-1}\right)}$ & $\mathbf{A U C}(\mathbf{n g ~ h} / \mathbf{m L})$ \\
\hline Fe-SLNs & $2.0 \pm 0.3$ & $330.51 \pm 15.18$ & $2,415 \pm 135$ & $0.06 \pm 0.02$ & $2,912 \pm 164$ \\
Iron marketed tablet & $0.75 \pm 0.2$ & $90.22 \pm 8.21$ & $695 \pm 61$ & $0.30 \pm 0.12$ & $756 \pm 81$ \\
\hline
\end{tabular}

Note: The significance level was set at $P<0.05$.

Abbreviations: Fe-SLNs, iron solid lipid nanoparticles; $C_{\max }$, maximum plasma concentration; $T_{\max }$, maximum plasma level.

Poloxamer 188 is a surfactant facilitating emulsification and formed more rigid surfactant films and therefore improved the stability. ${ }^{38}$ The small particle size obtained with Poloxamer 188 as surfactants could be due to its containing lipophilic chains that might be responsible for fine emulsification of hot solid lipid with water. ${ }^{39}$

The SC had a huge effect on particle size. By increasing its concentration, the size was decreased. At a low SC $(0.5 \%$ and $1 \%)$, particles in the nanometer range could not be generated. This could be due to the amount of Poloxamer 188 being used in these formulae not being adequate to provide sufficient coverage of the new surfaces generated due to particle size reduction during the ultrasonication step. This, in turn, results in reduced stabilization of the particle dispersion, and, as a result, a higher SC is essential to prevent particle aggregation. ${ }^{40}$ Higher concentration of surfactant (3\%) is expected to reduce surface tension resulting in reduction of the particle size, but further increases in SC (4\%) may not significantly affect the particle size once the optimum packing is reached. However, the decrease in size in this case was due to the reduction in entrapment efficiency. Liu et al also reported similar results of decreased particle size with reduction in entrapment efficiency. ${ }^{41}$ Increasing the SC led to an increase in $\mathrm{EE} \%$ independent of the type and concentration of lipids. However, when the concentration was increased above a certain level, the entrapment efficiency was decreased, which could be explained by the partitioning phenomenon, according to which at a high SC level in the external phase, the solubility of the drug in the external phase increases, thereby increasing the partitioning of the drug from the internal to the external phase of the medium and leading to a decrease in the entrapment efficiency. ${ }^{42}$

Homogenization followed by ultrasonication is a reliable, simple, and successful method for preparing uniform Fe-SLNs and homogenous in appearance. During preparation, the HT has a small effect on particle size after a certain time (3 minutes), which may be expected as the homogenization step is only utilized for emulsification of the lipid in the aqueous phase, and this step does not produce the final NPs. By increasing the HT time by more than 3 minutes, no further reduction in particle size was observed. The UT had a huge effect on the particle size; particle size was dramatically decreased as the sonication time increased. This could be due to the fact that sonication was responsible for making the final particle size in SLNs, which broke the coarse emulsion drops into nanoemulsion droplets. ${ }^{43}$ Hosny and Aljeaid have also reported that the particle size of SLNs was dependent on the sonication time. ${ }^{44}$

Because SLNs with low zeta potential are unstable and highly susceptible to aggregation on storage, charge inducing agents were added during the preparation of SLNs. This charge prevented particle aggregation and changes in particle size. ${ }^{45}$

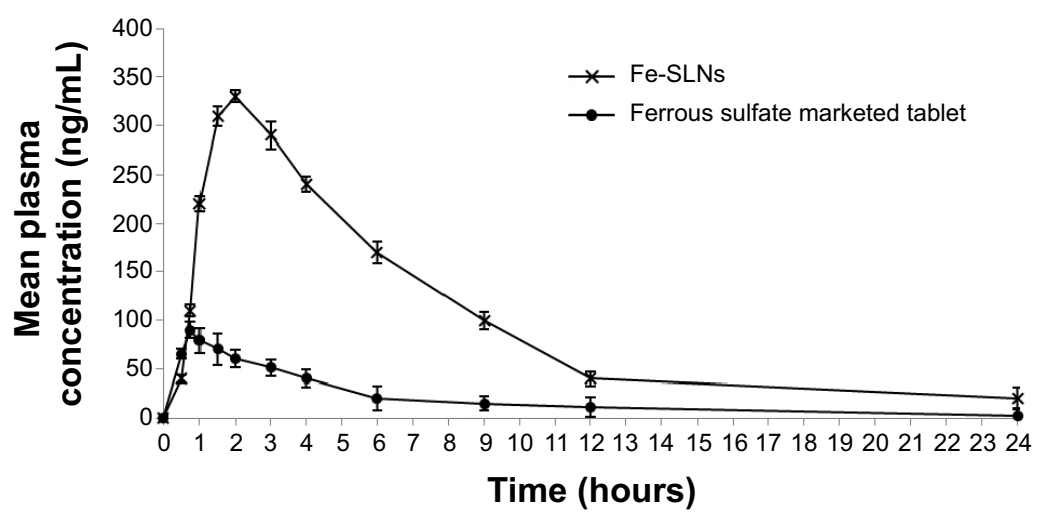

Figure 2 Plasma concentration versus time curve of iron measurements after oral administration of Fe-SLNs and marketed tablets to rabbits at the dose of 10 mg/kg. Abbreviation: Fe-SLNs, iron solid lipid nanoparticles. 
From the results discussed above, the optimum formulation conditions for preparation of Fe-SLNs were homogenization for 3 minutes followed by ultrasonication for 3 minutes for SLNs prepared from 3\% Compritol 888 ATO, $1 \%$ lecithin, 3\% Poloxamer 188, and 0.2\% DHP. These formulae were prepared and subjected to in vivo pharmacokinetic study.

The pharmacokinetic study of Fe-SLNs showed lower initial plasma concentration than that of the tablet for the first 30 minutes, possibly because iron was released slowly from SLNs for an extended period. After 30 minutes, the plasma concentration of iron in case of Fe-SLNs started to rise faster than that of the tablet. The $T_{\max }$ of iron from Fe-SLN was $330 \mathrm{ng} / \mathrm{mL}$, while in case of tablet the $C_{\max }$ was only $90 \mathrm{ng} /$ $\mathrm{mL}$. This result indicated that the formulation of iron as Fe-SLN enhanced its absorption and permeation character across the membrane of the GIT. The results also revealed that Fe-SLNs can significantly modify the pharmacokinetic profile and can increase the drugs bioavailability by more than fourfold in comparison with the marketed oral tablet formulation. This was due to the fact that iron is a drug with poor aqueous solubility, and the preparation of this drug as a SLNs enhanced not only its solubility but also tissue permeability. Also, the pharmacokinetic characteristics of drugs upon delivery in SLN formulation are dictated by the properties of the SLNs rather than by the physicochemical properties of the drug molecules. Additionally, the presence of Poloxamer 188 surfactant in the SLN formula causes a steric hindrance that helps in reducing the tissue uptake by evading the reticuloendothelial system (RES), which will increase the residence time of SLNs in the blood circulation. In addition, it has been reported that absorption of SLNs occurs mainly through the lymphatic uptake pathway by $\mathrm{M}$ cells of the Peyer's patches ${ }^{46}$ which exhibited a clear dependency on the particle characteristics such as size and surface properties.

\section{Conclusion}

The formulation of iron as oral SLNs, which constitutes a novel drug delivery system, enhanced the bioavailability by more than fourfold compared to the marketed tablet. The optimum Fe-SLN formula was composed of 3\% Compritol 888 ATO, $1 \%$ lecithin, $3 \%$ Poloxamer 188, and $0.2 \%$ DHP. The use of this formula could eliminate the major drawbacks of the conventionally used tablet. Of course, it will not obviate the need for further clinical evaluation regarding the bioactivity and toxicity for this novel formula, which may provide clinicians with other important data.

\section{Acknowledgment}

This Project was funded by the Deanship of Scientific Research (DSR), King Abdulaziz University, Jeddah, under grant No. (98/166/1434). The authors, therefore, acknowledge with thanks DSR technical and financial support.

\section{Disclosure}

The authors report no conflicts of interest in this work.

\section{References}

1. Brady PG. Iron deficiency anemia: a call for South. Med J. 2007;100: 966-967.

2. Conrad ME, Umbreit JN. A concise review: iron absorption - the mucin - mobilferrin - integrin pathway. A competitive pathway for metal absorption. Am J Hematol. 1993;42:67-73.

3. WHO global database on child growth and malnutrition. Geneva:World Health Organization; 2002.

4. Calis JC, Phiri KS, Faragher EB, et al. Severe anemia in Malawian children. N Engl J Med. 2008;358:888-899.

5. Dreyfuss ML, Stoltzfus RJ, Shrestha JB, et al. Hookworms, malaria and vitamin A deficiency contribute to anemia and iron deficiency among pregnant women in the plains of Nepal. J Nutr. 2000;130:2527-2536.

6. Arie H. Methodological Choices for Calculating the Disease Burden and Cost-of-Illness of Foodborne Zoonoses in European Countries. The Netherlands: Med Vet Net; 2007.

7. Park YW, Mahoney AW, Hendricks DG. Bioavailability of different sources of ferrous sulfate iron fed to anemic rats. J Nutr. $1983 ; 113: 2223-2228$.

8. Auerbach M, Liang AS, Glaspy J. Intravenous iron in chemotherapy and cancer-related anemia. Community Oncol. 2012;9:289-295.

9. Bregman DB, Morris D, Koch TA, He A, Goodnough LT. Hepcidin levels predict nonresponsiveness to oral iron therapy in patients with iron deficiency anemia. Am J Hematol. 2013;88:97-101.

10. Fritz JC, Pia GW, Roberts T, Boehne JW, Hove EL. Biological availability in animals of iron from common dietary sources. J Agric Food Chem. 1970;18:647-651.

11. Farmer BR, Mahoney AW, Hendricks DG, Gillett T. Iron bioavailability of hand-deboned and mechanically deboned beef. J Food Sci. 1977;42:1630-1632.

12. Hanaa E, Al Sayed A, Eman A, Kawkab A. Treatment of anemia progression via magnetite and folate nanoparticles in vivo. ISRN Nanotechnol. 2014;2014:1-13.

13. Liudmyla R, Andrii D, Tamara G. Iron nanoparticles effectiveness for new antianemic preparations development. Nano Conf. 2013;10:16-18.

14. Pasricha SR, Drakesmith H, Black J, Hipgrave D, Biggs BA. Control of iron deficiency anemia in low- and middle income countries. Blood. 2013;121:2607-2617.

15. Wissing SA, Kayser O, Müller RH. Solid lipid nanoparticles for parenteral drug delivery. Adv Drug Deliv Rev. 2004;56(9):1257-1272.

16. Cavalli R, Gasco MR, Chetoni P, Burgalassi S, Saettone MF. Solid lipid nanoparticles (SLN) as ocular delivery system for tobramycin. Int J Pharm. 2002;238:241-245.

17. Schwarz C, Mehnert W. Solid lipid nanoparticles (SLN) for controlled drug delivery II. Drug incorporation and physicochemical characterization. J Microencapsul. 1999;16(2):205-213.

18. Dingler A, Gohla S. Production of solid lipid nanoparticles (SLN): scaling up feasibilities. J Microencapsul. 2002;19(1):11-16.

19. Luo Y, Chen D, Ren L, Zhao X, Qin J. Solid lipid nanoparticles for enhancing vinpocetine's oral bioavailability. J Controlled Release. 2006;114(1):53-59. 
20. Zhang N, Ping Q, Huang G, Xu W, Cheng Y, Han X. Lectin-modified solid lipid nanoparticles as carriers for oral administration of insulin. Int J Pharm. 2006;327:153-159.

21. Göppert TM, Müller RH. Adsorption kinetics of plasma proteins on solid lipid nanoparticles for drug targeting. Int $J$ Pharm. 2005;302:172-186.

22. Kamble S, Vaidya K, Bhosale V, Chaudhar PD. Solid lipid nanoparticles and nanostructured lipid carriers - an overview. Int J Pharm Chem Biol Sci. 2012;2(4):681-691.

23. Ekambaram P, Sathali H, Priyanka K. Solid lipid nanoparticles: a review. Sci Rev Chem Commun. 2012;2(1):82-85.

24. Sinha VR, Srivastava S, Goel H, Jindal V. Solid lipid nanoparticles (SLN's) - trends and implications in drug targeting. Int J Adv Pharm Sci. 2010;1:212-238.

25. Cortesi R, Esposjto E, Luca G, Nastruzzi C. Production of lipospheres as carriers for bioactive compounds. Biomaterials. 2002;23(11):2283-2294.

26. Song C, Liu S. A new healthy sunscreen system for human: solid lipid nannoparticles as carrier for 3,4,5-trimethoxybenzoylchitin and the improvement by adding vitamin E. Int J Biol Macromol. 2005;36:116-119.

27. Ahlin P, Kristl J, Smid-Kobar J. Optimization of procedure parameters and physical stability of solid lipid nanoparticles in dispersions. Acta Pharm. 1998;48:257-267.

28. Chen CC, Tsai TH, Huang ZR, Fang JY. Effects of lipophilic emulsifiers on the oral administration of lovastatin from nanostructured lipid carriers: physicochemical characterization and pharmacokinetics. Eur J Pharm Biopharm. 2010;74:474-482.

29. Elshafeey A, Bendas E, Mohamed O. Intranasal microemulsion of sildenafil citrate: in vitro evaluation and in vivo pharmacokinetic study in rabbits. AAPS Pharm Sci Tech. 2009;10(2):361-367.

30. Dias NC, Rustum AM. Development and validation of an HPLC stability-indicating method for identification and assay of elemental iron(II) in pharmaceutical drug products using reversed-phase HPLC. J AOAC Int. 2011;94(4):1233-1239.

31. Meyyanathan SN, Muralidharan S, Rajan S, Gopal K, Suresh B. A simple sample preparation with HPLC-UV method for estimation of amlodipine from plasma: application to bioequivalence study. Open Chem Biomed Meth J. 2008;1:22-27.

32. Yang Z, Gao S, Wang J, et al. Enhancement of oral bioavailability of 20(S)-ginsenoside Rh2 through improved understanding of its absorptionand efflux mechanisms. Drug Metab Dispos. 2011;39:1866-1872.

33. Battaglia L, Trotta M, Gallarate M, Carlotti ME, Zara GP, Bargoni A. Solid lipid nanoparticles formed by solvent-in-water emulsion diffusion technique: development and influence on insulin stability. J Microencapsul. 2007;24:672-684.
34. Sanna V, Caria G, Mariani A. Effect of lipid nanoparticles containing fatty alcohols having different chain length on the ex vivo skin permeability of econazole nitrate. Powder Technol. 2010;201:32-36.

35. Shah KA, Date AA, Joshi MD, Patravale VB. Solid lipid nanoparticles (SLN) of tretinoin: potential in topical delivery. Int $J$ Pharm. 2007;345:163-171.

36. Tanvir S, Qiao L. Surface tension of nanofluid-type fuels containing suspended nanomaterials. Nanoscale Res Lett. 2012;7:226.

37. Uner M, Wissing SA, Yener G, Müller RH. Influence of surfactants on the physical stability of solid lipid nanoparticle (SLN) formulations. Pharmazie. 2004;59:331-332.

38. Westesen K, Siekmann B, Koch MHJ. Investigations on the physical state of lipid nanoparticles by synchrotron X-ray diffraction. Int $J$ Pharm. 1993;93:189-199.

39. Das S, Ng WK, Tan RBH. Are nanostructured lipid carriers (NLCs) better than solid lipid nanoparticles (SLNs): development, characterizations and comparative evaluations of clotrimazole-loaded SLNs and NLCs? Eur J Pharm Sci. 2012;47:139-151.

40. Yassin AE, Anwar MK, Mowafy HA, El-Bagory IM, Bayomi MA, Alsarra IA. Optimization of 5-fluorouracil solid-lipid nanoparticles: a preliminary study to treat colon cancer. Int J Med Sci. 2010;7:398-408.

41. Liu J, Gong T, Wang C, Zhong Z, Zhang Z. Solid lipid nanoparticles loaded with insulin by sodium cholate-phosphatidylcholine-based mixed micelles: preparation and characterization. Int $J$ Pharm. 2007;340:153-162.

42. Yang Q, Owusu-Ababio G. Biodegradable progesterone microsphere delivery system for osteoporosis therapy. Drug Dev Ind Pharm. 2000;26:61-70.

43. Surajit D, Wai K, Parijat K, Kim S, Tan RB. Formulation design, preparation and physicochemical characterizations of solid lipid nanoparticles containing a hydrophobic drug: effects of process variables. Colloids Surf B Biointerfaces. 2011;88:483-489.

44. Hosny K, Aljeaid B. Sildenafil citrate as oral solid lipid nanoparticles: a novel formula with higher bioavailability and sustained action for treatment of erectile dysfunction. Expert Opin Drug Deliv. 2014;11(7):1015-1022.

45. Doijad C, Marvi V, Deshmukh V. Formulation and targeting efficiency of cisplatin engineered solid lipid nanoparticles. Int J Pharm. 2008;70:203-207.

46. Araujo L, Sheppard M, Löbenberg R, Kreuter J. Uptake of PMMA nanoparticles from the gastrointestinal tract after oral administration to rats: modification of the body distribution after suspension in surfactant solutions and in oil vehicles. Int J Pharm. 1999;176:209-224.
Drug Design, Development and Therapy

\section{Publish your work in this journal}

Drug Design, Development and Therapy is an international, peerreviewed open-access journal that spans the spectrum of drug design and development through to clinical applications. Clinical outcomes, patient safety, and programs for the development and effective, safe, and sustained use of medicines are a feature of the journal, which
Dovepress

has also been accepted for indexing on PubMed Central. The manuscript management system is completely online and includes a very quick and fair peer-review system, which is all easy to use. Visit http://www.dovepress.com/testimonials.php to read real quotes from published authors. 